\title{
Newly Developed Systems for the Magnetostrictive Materials Strain Measurements
}

\author{
K. KuCZyŃski, A. Bieńkowski \\ Institute of Metrology and Measurement Systems \\ Warsaw University of Technology \\ Sw. A. Boboli 8, 02-525 Warsaw, Poland \\ L. Salbut and G. Dymny \\ Institute of Micromechanics and Photonics \\ Warsaw University of Technology \\ Sw. A. Boboli 8, 02-525 Warsaw, Poland
}

\begin{abstract}
Magnetostrictive effect is connected with changes of the dimension of soft magnetic materials during the process of theirs magnetization. The paper presents newly developed measuring systems for testing of the strain distribution in the magnetostrictive materials. The system uses simultaneously two measurement techniques: strain-gauge sensor for local strain measurement and grating interferometry for the global determination of strain distribution.
\end{abstract}

PACS numbers: 75.80.+q, 42.50.St, 07.10.Pz, 07.60.Ly

\section{Introduction}

Magnetostrictive materials are an important branch of the materials tree for several reasons into applications within the aerospace, military, leisure, and industrial areas. Different methodologies of the measurement of the magnetostriction were described in previous paper [1,2]. The paper presents systems using simultaneously two measurement techniques: strain-gauge sensors for local strain measurement and optical: grating interferometry for in-plane displacement/strain distribution determination and two-beams interferometry for out of plane and total elongation of the specimen under test.

\section{Strain-gauge technique}

The schematic block diagram of the installation utilizing semiconductor strain-gauge measuring techniques is given in Fig. 1. This solution creates pos- 
sibility of simultaneous measurement of the magnetistriction $\lambda$ in the function of magnetizing field $H$. Moreover, developed measuring installation gives possibility of testing of the initial and the reversal hysteresis $\lambda(H)$ loops. As a result it gives more complete information about magnetostrictive properties of tested materials, than known methods of measurement saturation magnetostriction $\lambda_{\mathrm{S}}$. In addition, simultaneous measurements of $\lambda$ and $H$ give more complete information on process of the magnetization of the soft magnetic materials.

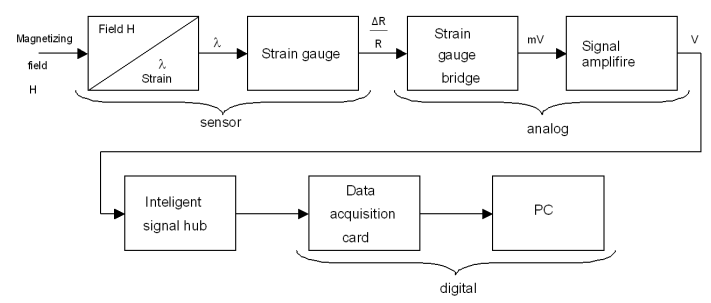

Fig. 1. Schematic of measuring system.

\section{Interferometry}

Grating (moiré) interferometry (GI) is an experimental method to determine the components of displacement $(u, v)$ or strain $\left(\varepsilon_{x}, \varepsilon_{y}\right)[3,4]$. On a specimen subjected to analysis, a high frequency grating of equidistant lines is deposited. When the specimen is subjected to stresses, deformation of the specimen, and consequently of the grating applied to it, occurs. The deformed grating is then symmetrically illuminated by two mutually coherent beams with plane wave fronts. The incident angle of these beams is equal to the first diffraction order angle of specimen grating. In such configuration +1 and -1 order beams propagate coaxially along the grating normal and interfere. Wave fronts of these beams are now not plane due to specimen grating deformation and the intensity distribution of the interferogram becomes

$$
I(x, y)=a(x, y)+b(x, y) \cos \left(\frac{4 \pi}{p} u(x, y)\right)
$$

where $a(x, y)$ and $b(x, y)$ are the local values of background and contrast in an interferogram, $u(x, y)$ represents in-plane displacements vector in direction perpendicular to the grating lines and $p$ is the grating period.

GI is insensitive to out-of-plane displacement, so if the information about it is required other type of interferometers should be used. The most popular are classical interferometers working in Fizeau or Twyman-Green configurations [4]. In this case the intensity distribution can be expressed as follows:

$$
I(x, y)=a(x, y)+b(x, y) \cos \left(\frac{2 \pi}{\lambda} w(x, y)\right),
$$

where $\lambda$ is the wavelength of illuminating beam and $w(x, y)$ represents out-of-plane 
displacement. These interferometers are insensitive to in-plane displacement, so both grating and classical interferometric techniques, can be used simultaneously. For testing of magnetostrictive effect we propose to use the grating interferometer for in-plane displacement distribution measurement at local area combined with Fizeau interferometer for measurement of total specimen elongation. The idea of these systems is shown in Fig. 2.
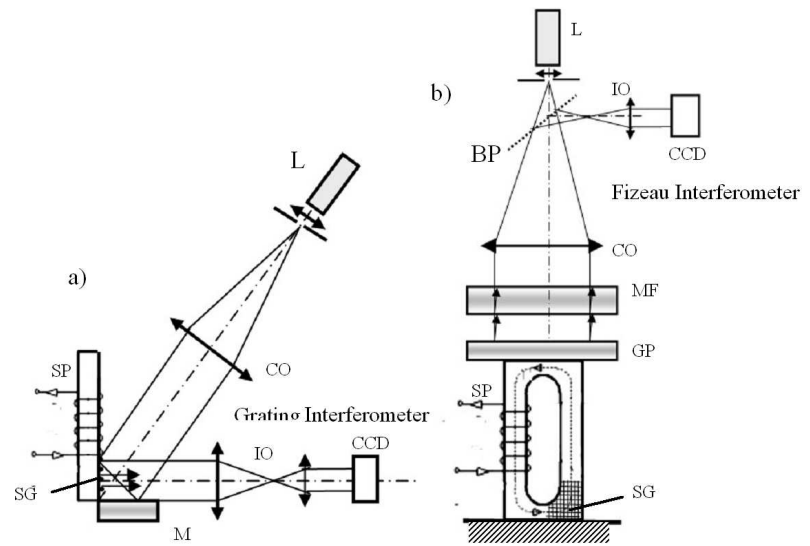

Fig. 2. Schemes of grating interferometer (a) for in-plane displacement and Fizeau interferometer (b) for specimen elongation measuremens. L - laser, CO - collimating objective, IO — imaging optics, BP — beam splitter, MF — master flat, GP — glass plate, $\mathrm{M}$ - mirror, $\mathrm{SP}$ - specimen under test, $\mathrm{SG}$ - specimen grating.

On the specimen side surface the specimen diffraction grating SG with frequency 1200 lines $/ \mathrm{mm}$ is attached. Perpendicularly to this surface the mirror M is placed. This configuration enables symmetrical illumination of the specimen grating SG required by GI method. The small glass plate GP is glued on the top surface. It should be used because the specimen surface has not optical quality. Using the Fizeau interferometer, the position of this plate, depending on specimen elongation, is measured. The results of in-plane displacement and strain distribution on side surface and out-of-plane displacement of top surface (in this case equal to specimen elongation) are presented at the next part of the paper.

\section{Results of investigation}

Figure 3a. and 3b. present example of in-plane displacements in y-direction and total elongation using grating and Fizeau interferometers. The initial curve and the magnetostrictive hysteresis loop using semiconductor strain- gauge measuring techniques is given in Fig. 3c. 


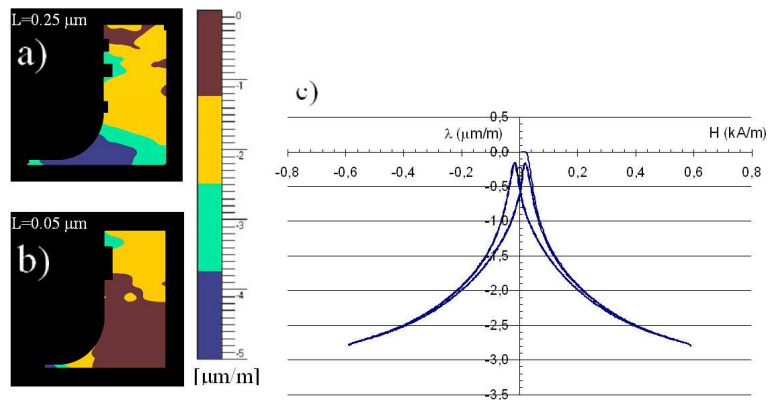

Fig. 3. Example of in-plane displacements in $y$-direction and total elongation $(\Delta L)$ measurements (a) in magnetic saturation, (b) in magnetic remanence - using grating and Fizeau interferometers; (c) the initial curve and the magnetostrictive hysteresis loop of the $\left(\mathrm{Fe}_{2} \mathrm{O}_{3}\right)_{50}(\mathrm{NiO})_{17.5}(\mathrm{ZnO})_{32}(\mathrm{CoO})_{0.5}$ ferrite using semiconductor strain-gauge measuring techniques [5].

\section{Conclusion}

Application of two interferometric methods combined with tensometric technique for testing of magnetostrictive properties is presented. GI is one of the most useful optical methods for in-plane displacement distribution measurements in the local field of view (in our case $15 \mathrm{~mm} \times 15 \mathrm{~mm}$ ). For measurement of total elongation of the sample, the classical Fizeau interferometer is proposed. Displacement or strain maps and elongation measured at different point of magnetostrictive hysteresis gives information about influence of specimen geometry and its material inhomogeneity on the magnetisation process. Measuring installation utilizes semiconductor strain-gauge measuring techniques [5]. This solution creates possibility of simultaneous measurement of the magnetistriction $\lambda$ in the function of magnetizing field $H$. Moreover developed measuring installation gives possibility of testing of the initial and the reversal hysteresis $\lambda(H)$ loops.

\section{References}

[1] R. O'Handley, Modern Magnetic Materials: Principles and Applications, Wiley, New York 2000.

[2] G. Vlasák, J. Magn. Magn. Mater. 215-216, 479 (2000).

[3] D. Post, B. Han, P. Ifju, High Sensitivity Moiré, Springer-Verlag, New York 1994.

[4] K. Patorski, M. Kujawiska, L. Salbut, Interferometria Laserowa z Automatyczna Analiza Obrazu, Oficyna Wydawnicza Politechniki Warszawskiej, Warszawa 2005.

[5] K. Kuczyński, A. Bieńkowski, R. Szewczyk, in: Proc. 14th IMEKO TC4 Symp., Eds. J. Mindykowski, T. Tarasiuk, Gdynia Maritime University, Gdynia/Jurata 2005, p. 434. 